\title{
Elaboration de plateformes biomimétiques à base de dopamine pour la fonctionnalisation du titane
}

\section{Elaboration of biomimetic dopamine platforms for the functionalization of titanium surfaces}

\author{
William Laure, Cédric Zobrist, Joël Lyskawa, David Fournier et Patrice Woisel \\ Unité Matériaux et Transformations (UMET) UMR CNRS 8207, Université de Lille 1, France
}

\begin{abstract}
The aim of this study concerns the functionalization of titanium surfaces by using versatile dopamine based on biomimetic anchors. This general concept was exploited to design new biomaterials which might be used as medical implants. In this context, two different grafting strategies based onto the "grafting to" approach were developed. The first one consisted on the synthesis and the immobilization of well-defined catechol end-functionalized polymers onto titanium surfaces. The second strategy was based on the functionalization of titanium surfaces by using dopamine derivates containing, on the one hand, a catechol unit for surface attachment and, on the other hand, a furan or a maleimide moiety able to react, on demand, through reversible Diels Alder (DA) reaction.
\end{abstract}

\begin{abstract}
Résumé. L'étude concerne la fonctionnalisation de surfaces à base de titane à partir d'une ancre chimique biomimétique modifiable chimiquement, la dopamine, dans le but de concevoir des biomatériaux utilisables en tant que dispositifs implantables. Deux approches différentes de greffage par la stratégie 《graft to» sont proposées. La première consiste en la synthèse et l'immobilisation sur les surfaces de titane de polymères parfaitement définis et stimulables fonctionnalisés par un motif catéchol en extrémité de chaîne. La seconde propose de modifier préalablement la surface par une ancre chimique spécifique intégrant un motif catéchol d'une part et un motif furane ou maléimide d'autre part permettant d'engager sur demande une réaction réversible de cycloaddition de type Diels Alder (DA) avec une large gamme de molécules ou polymères fonctionnalisés par l'entité complémentaire.
\end{abstract}

\section{INTRODUCTION}

Au cours des dernières décennies, les biomatériaux à base de titane ont rencontré un fort engouement dans la conception de prothèses de part leurs propriétés mécaniques, thermiques et surtout leur biocompatibilité ${ }^{1}$. L'intégration tissulaire d'un implant ou d'une prothèse est dirigée par de multiples facteurs provenant du comportement physicochimique du matériau. Ainsi, de nos jours, dans le domaine des dispositifs médicaux implantables, la tendance dans la recherche internationale n'est plus de remplacer des organes par des biomatériaux inertes mais de proposer des biomatériaux dits «intelligents» et spécifiquement adaptés au site d'implantation afin de lutter contre des complications irréversibles qui peuvent conduire, dans les cas les plus graves, au décès du patient. Dans cette optique, le greffage de polymères parfaitement définis pouvant contenir potentiellement des molécules bioactives sur des surfaces de titane semble être une technique efficace et adaptée.

Parmi les différentes techniques de modification de surfaces répertoriées dans la littérature [2,3], l'utilisation de dérivés de catéchols semble être celle présentant le plus fort potentiel, notamment en raison de ses fortes propriétés d'adhésion sur de nombreux supports [4]. La dopamine, une molécule commerciale dérivée des catéchols, possède une fonction amine primaire susceptible d'être modifiée chimiquement permettant d'apporter une fonctionnalité supplémentaire.
Le but de cette étude consiste à immobiliser des molécules ou polymères fonctionnels sur la surface d'un substrat à base de titane, par une accroche solide par l'intermédiaire d'une plateforme fonctionnalisable à base de dopamine [5]. Deux approches ont été élaborées : la première repose sur le greffage de polymères parfaitement définis intégrant en extrémité de chaîne un motif catéchol et la seconde approche consiste à fonctionnaliser préalablement la surface à l'aide d'une ancre spécifique comportant d'une part le motif catéchol et d'autre part une entité susceptible de réagir avec une entité complémentaire dans le cadre d'une réaction réversible de cycloaddition de type Diels Alder [6].

\section{PARTIE EXPÉRIMENTALE}

Dans un premier temps, nous avons élaboré et synthétisé une large gamme de polymères de type poly- $t$-butyl acrylate, poly- $N$-isopropylacrylamide et polystyrène par le processus de polymérisation radicalaire contrôlée par transfert de chaîne réversible par addition-fragmentaion (RAFT) en utilisant un agent de transfert fonctionnalisé en extrémité de chaîne par un motif catéchol. Des contrôles de la cinétique de polymérisation et de l'évolution des masses molaires ont été effectués et montrent le caractère vivant de la polymérisation. Les polymères fonctionnalisés ont été caractérisés par RMN, UV-visible et GPC. L'immobilisation des polymères sur les surfaces de titane 

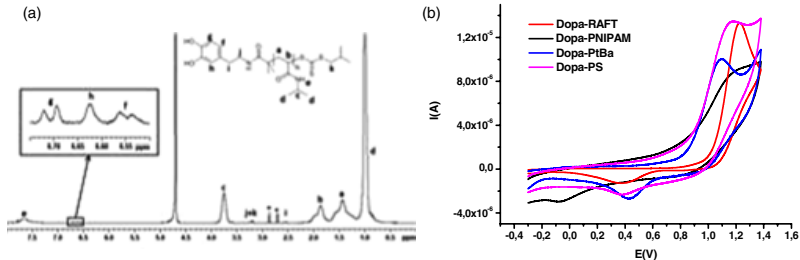

Figure 1. a) Spectre $\mathrm{RMN}{ }^{1} \mathrm{H}$ de la dopamine-PNIPAM $\left(300 \mathrm{MHz}, \mathrm{D}_{2} \mathrm{O}\right)$ et $\left.\mathrm{b}\right)$ voltampérogrammes cycliques de Dopa RAFT, Dopa-PNIPAM, Dopa-Pt-BA and Dopa-PStyrène.

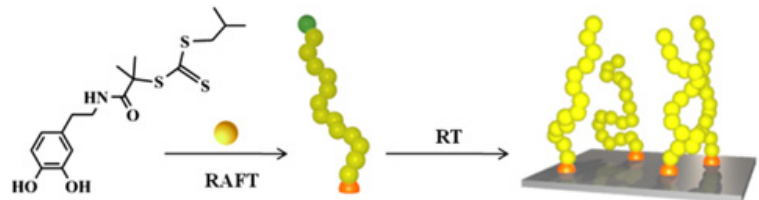

Schéma 1. Illustration de fonctionnalisation de surfaces de titane par des polymères élaborés à partir d'un agent de transfert intégrant le motif catéchol.

a ensuite été suivie par SPR, XPS, AFM et par mesures d'angle de contact.

Dans un second temps, nous avons synthétisé deux ancres chimiques intégrant chacune le motif catéchol à une extrémité et une entité furane ou maléimide à l'autre extrémité dans le but d'engager une réaction de cycloaddition de type Diels Alder avec une molécule ou polymère modifié par l'entité complémentaire. Les ancres chimiques ainsi que les entités fonctionnalisées mises en jeu lors des étapes de DA ont été caractérisées par RMN, $\mathrm{CV}$ et GPC. Le greffage des ancres chimiques et les étapes réversibles d'immobilisation des molécules fonctionnelles ont été suivis par CV, XPS et angles de contact.

\section{RÉSULTATS ET DISCUSSION}

Fonctionnalisation en 1 étape :

Nous avons élaboré un agent de transfert de type RAFT comportant à une extrémité le motif catéchol pouvant se greffer sur la surface de titane et à l'autre extrémité un groupement trithiocarbonate permettant de générer une polymérisation radicalaire contrôlée (schéma 1).

Plusieurs polymérisations ont été effectuées en utilisant l'agent de transfert Dopa RAFT préalablement caractérisé par RMN et UV-visible. Le poly $N$-isopropylacrylamide (PNIPAM), le polybutyl acrylate (Pt-BA) ou encore le polystyrène présentant en extrémité de chaîne le motif catéchol ont été obtenus en présence d'un amorceur radicalaire (AIBN) dans la DMF et ont été caractérisés par RMN ${ }^{1} \mathrm{H}$ (Fig. 1a) et GPC. La présence du motif catéchol a quant à elle été démontrée par des mesures de $\mathrm{CV}$ dans l'acétonitrile (Fig. 1b).

Le greffage des polymères a été réalisé en immergeant les surfaces de titane dans une solution de polymères fonctionnalisés à $1 \mathrm{mM}$ pendant une nuit à température ambiante et a été caractérisé par des mesures de résonance plasmonique de surface (SPR), CV, XPS, AFM et angle de contact.

Fonctionnalisation en 2 étapes :

Nous avons tout d'abord synthétisé deux nouvelles ancres chimiques incorporant d'une part un motif catéchol

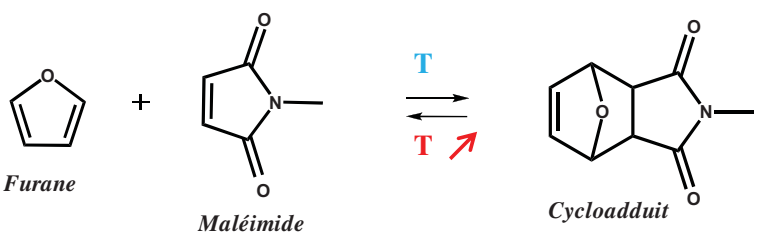

Schéma 2. Représentation de la réaction thermoréversible de type Diels Alder.
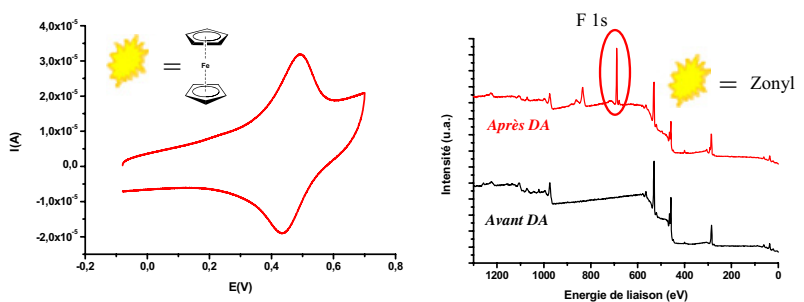

Figure 2. Voltampérogramme cyclique d'une surface modifiée avec l'entité électro-active après réaction de DA (gauche) et b) spectres XPS de surfaces modifiées avec l'oligomère fluoré avant et après réaction de DA (droite).

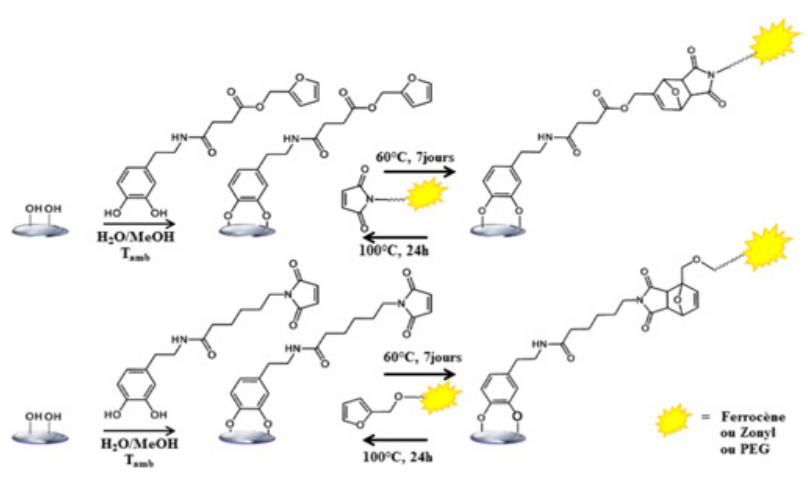

Schéma 3. Représentation de la fonctionnalisation de surfaces de titane par la combinaison de la plateforme dopamine avec la chimie de type «Diels Alder».

capable de former des liaisons fortes avec la surface du substrat et d'autre part une entité furane ou maléimide pouvant donner lieu à une réaction de cycloaddition [ $4+$ 2] thermoréversible plus connue sous le nom de réaction de Diels Alder (schéma 2).

Les ancres dopamine furane (DF) et dopamine maléimide (DM) synthétisées ont été caractérisées par $\mathrm{RMN}{ }^{1} \mathrm{H}$ et ${ }^{13} \mathrm{C}$ et par CV. La fonctionnalisation des surfaces de titane par l'intermédiaire de ces dérivés de dopamine modifiés a été effectuée par une simple immersion dans des solutions d'ancres à $1 \mathrm{mM}$ pendant une nuit sous agitation et a été confirmée par des mesures de voltampérométrie cyclique.

La plateforme de fonctionnalisation de surface obtenue grâce à ce système permet ainsi de greffer une large gamme de réactifs par l'intermédiaire de la réaction de cycloaddition de Diels Alder. Nous avons alors synthétisé plusieurs molécules sondes fonctionnalisées : une première possédant un motif électro-actif (ferrocène), une seconde composée d'un oligomère fluoré (Zonyl) et enfin une dernière constituée d'un polymère à caractère hydrophile (PEG) (schéma 3). 
Les sondes fonctionnalisées ont été caractérisées par RMN ${ }^{1} \mathrm{H}, \mathrm{CV}$, IR, GPC puis engagées dans la réaction de DA avec les supports modifiés pendant une semaine à $60^{\circ} \mathrm{C}$. La réaction de rétro $\mathrm{DA}(\mathrm{rDA})$ a été, quant à elle, réalisée en immergeant les échantillons dans du toluène à $100^{\circ} \mathrm{C}$ pendant $24 \mathrm{~h}$. L'immobilisation des molécules sondes sur les surfaces et le caractère réversible de cette réaction ont été démontrés et caractérisés par CV, XPS et mesures d'angle de contact (Fig. 2).

\section{CONCLUSION}

Une première stratégie a permis de greffer sur des surfaces de titane des polymères parfaitement définis obtenus par polymérisation radicalaire contrôlée et modifiés en extrémité de chaîne par une unité dopamine. Une seconde a donné lieu à la réalisation d'une plateforme de fonctionnalisation de surface à l'aide d'ancres spécifiques susceptibles de réagir réversiblement avec des entités diverses et variées dans le cadre d'une réaction de cycloaddition de type Diels Alder. L'étude proposée permet d'envisager la possibilité de modifier de nombreuses surfaces à base de titane grâce au caractère versatile de l'ancre dopamine et d'entrevoir son utilisation dans de multiples applications telles que la modification de surfaces d'implants médicaux.

\section{Références}

[1] Textor M et al., Bio-Implant Interface, 2003, 341.

[2] Schwartz J. et al., Materials Science and Engineering: C 2003, 23 (3), 395-400.

[3] Ojamäe L. et al., J. Col. Int. Sci. 2006, 296 (1), 71-78.

[4] Lee H. et al., Science, 2007, 318, 426-430.

[5] Zobrist, C. et al., Macromol., 2011, 44(15), 58835892.

[6] Dirlam P.T. et al., Langmuir, 2009, 26(6), 3942-3948. 